\section{"Cope's Sign" and Reflex Bradycardia in Two Patients with Cholecystitis}

\author{
MICHAEL V. O'REILLY \\ MARTIN J. KRAUTHAMER
}

\section{British Medical fournal, 1971, 2, 146}

Sir Zachary Cope (1970) recently suffered an attack of acute cholecystitis. Until noting a palpable gall bladder he initially thought the symptoms might be due to coronary ischaemia, especially since over the preceding six months he had experienced a number of attacks of perspiration accompanied by tachycardia. This confusing presentation and the wellknown association between the two diseases stimulated him to record for the first time some of the earliest manifestations of cholecystitis-namely, heavy epigastric/central chest pain accompanied by a palpable gall bladder. He noted that the initial symptoms may then abate for an interval only to be followed by the typical syndrome of severe right upper quadrant pain, etc.

We report two similar cases that were brought to our attention because of their simulation of a cardiac condition.

\section{Case 1}

Early on 28 November 1969 an 81-year-old man was admitted to hospital because of central lower chest discomfort and mild diaphoresis accompanied by some nausea but not by vomiting or hypotension. Physical examination showed a generally healthy man in no acute distress, with normal heart and lungs, except for a bradycardia of $56-60 / \mathrm{min}$ No abdominal masses or tenderness were noted; the electrocardiogram was normal. A provisional diagnosis of angina pectoris was entertained. After his evening meal he complained of nausea and mild right upper quadrant pain which did not radiate. Physical examination showed splinting of the muscles in the right upper quadrant and a poorly-defined mass. Heart rate was about 50 . He admitted to two similar attacks which subsided spontaneously over the previous 18 months. Next morning he was apyrexial, but a slightly raised white cell count and a shift to the left were noted. Liver function tests, serum lactate dehydrogenase, and E.C.G. remained normal. The abdominal splinting receded, the mass in the right upper quadrant disappeared, and his pulse stabilized at 70-80 without medication.

On 1 December a cholecystogram showed no gall-bladder function, but during that day the pain in the right upper quadrant recurred, became more severe, and was accompanied by vomiting of green fluid. No mass was palpable at this time; however, the usual therapy for acute cholecystitis was initiated (intravenous fluids, gastric suction, vagolytic drugs, and antibiotics). The symptoms became more severe and his gall bladder was removed the next morning (Dr. E. Tracey). Pathological examination showed cholelithiasis with chronic and acute necrotizing cholecystitis. $\mathrm{He}$ made an uneventful recovery.

Norwalk Hospital, Cardiac Laboratory, Norwalk, Connecticut, U.S.A MICHAEL V. O'REILLY, F.R.C.P.(C), M.R.C.P.I., Cardiologist. (Present address : Cardiopulmonary Department, Beth Israel Medical Centre,
New York 10003.)

MARTIN J. KRAUTHAMER, M.D., Cardiologist

\section{Case 2}

On 2 May 1970 a 56-year-old man complained of lower sternal and epigastric pain with vomiting of green fluid over the preceding six hours. He had been treated during the previous week for symptoms attributed to a renal tract infection. The pain, initially midline, became more intense and radiated to the right upper quadrant. Physical examination showed a somewhat pale and slightly diaphoretic man, otherwise not in distress, with normal heart and lungs, except for a pronounced (regular) bradycardia of $38-42$.

We saw the patient because the chest pain and bradycardia were considered compatible with an impending inferior-wall myocardial infarction. An E.C.G. was normal except for a sinus bradycardia of 38-42. There was a slightly tender mass in the right hypochondrium, palpation of which induced violent vomiting of nonbloody fluid. Though bowel sounds were infrequent there was no appreciable abdominal distention. The bradycardia responded well to intravenous atropine administered at regular intervals. The white count was slightly raised $(11,000)$, with a shift to the left (97\% polymorphs). Next day the abdominal signs abated and the bradycardia became intermittent. Liver function tests, serum lactate dehydrogenase, and E.C.G. were normal (and remained stable throughout his stay).

Though initially he had a slight pyrexia the abdominal signs and bradycardia cleared up almost completely over the next 36-48 hours, and the mass in the right upper quadrant disappeared. The provisional diagnosis remained acute cholechystitis. The pyrexia persisted, a cholecystogram showed non-function of the gall bladder, so he was put on intravenous fluids and antibiotics. Surgery was contemplated but postponed for a day owing to frequent premature ventricular contractions to allow an impending myocardial infarction to be ruled out. On the fifth day hi's gall bladder was removed (Dr. W. Kessler) under continuous E.C.G. monitoring. Except for intermittent premature ventricular contractions surgery was uncomplicated and the patient made an uneventful recovery. Pathological examination showed cholelithiasis, with chronic and acute haemorrhagic cholecystitis.

\section{Comment}

These two cases serve to emphasize a number of important features of gall-bladder disease. Firstly, that the initial symptoms (central epigastric/retrosternal pain and arrhythmias) may simulate coronary artery disease-this association has been critically reviewed by Friedman and In Hyg (1968). Careful history-taking and examination of the abdomen is mandatory in all cases, especially where the diagnosis and supporting laboratory investigations are conflicting or not clearcut. Secondly, an initially palpable gall bladder may later disappear and the symptoms subside without any therapy. The correct diagnosis may then be delayed and the patient treated as a case of coronary insufficiency, with possibly disastrous results. Thirdly, the reflex effects of gall-bladder (and intraabdominal) disease on the cardiovascular system can be misleading, especially if the palpable gall bladder is missed initially and then disappears. The serum bilirubin and serum enzymes may be normal in both conditions.

We wish to thank Dr. T. Bucky for permission to report a case under his care.

\section{References}

Cope, Z. (1970). British Medical fournal, 3, 147. Annals of Internal Med-
Friedman, G. D., and In Hyg, S. M. (1968). Annols icine, 68, 222 . 